\title{
MÉTHODE OPÉRATIONNELLE DE PRODUCTION D'ORTHOPHOTOS ET DE MNT DÉCIMÉTRIQUES À L'ÉCHELLE DU KILOMĖTRE CARRÉ PAR CERF-VOLANT
}

\author{
Denis Feurer ${ }^{1}$, Mohamed Amine El Maaoui², Mohamed Rached Boussema², Olivier Planchon ${ }^{1}$ \\ 1: IRD, UMR LISAH INRA-IRD-SupAgro, 2 place Viala, 34060 Montpellier, France \\ 2: ENIT, LTSIRS, B.P. 37, 1002 Tunis, Tunisie
}

\begin{abstract}
Résumé
Tous vecteurs confondus, ce sont les drones, notamment les multirotor, qui ont connu le plus fort développement pour l'acquisition d'images aériennes durant les cinq dernières années. L'imagerie accessible par ce type de plate-forme répond à un besoin de cartographie à une échelle intermédiaire entre l'imagerie submétrique (satellite à très haute résolution spatiale ou vecteurs aériens comme les ULM et certains drones) et l'imagerie acquise à très basse altitude à des résolutions parfois inférieures au centimètre. Par son prix élevé, l'imagerie submétrique aérienne et satellitaire laisse un grand nombre de besoins sans réponse acceptable. Parmi eux, citons l'imagerie à petit budget demandée par les collectivités locales ou les suivis denses (en particulier temporels). Finalement, la réglementation récente a encadré les vols drones de manière très contraignante, mettant virtuellement hors-la-loi tous les acteurs occasionnels d'imagerie par drone.

L'objectif de cette communication est de présenter une méthode simple, opérationnelle et à bas coût pour la prise de vue aérienne. II s'agit d'utiliser le cerf-volant comme vecteur alternatif aux aéronefs sans pilote. Le cerf-volant (avec le ballon) fait partie des premières plates-formes utilisées historiquement pour la photographie aérienne. II a connu un regain d'intérêt depuis les années 70-80 avec de nombreuses applications en archéologie et la disponibilité depuis les années 2000 de capteurs et logiciels abordables et performants le rend plus attractif encore. La méthode proposée ici permet la cartographie de superficies dépassant le kilomètre carré à une résolution décimétrique. Elle présente un certain nombre d'atouts, comme le coût modique de la plate-forme et la capacité de voler sur une très large plage de vents (de 5 à 40 $\mathrm{km} / \mathrm{h}$ ), un cerf-volant de six à dix mètres carrés ayant la même charge utile qu'un drone pour une autonomie supérieure. En revanche, la principale difficulté de mise en œuvre opérationnelle du cerf-volant pour la cartographie par image aérienne est le manque de contrôle du vecteur, ce qui pose quelques verrous au niveau de l'acquisition et du traitement des données.

Nous présentons dans cette communication les avancées méthodologiques réalisées afin de dépasser ces limites. Nous détaillons tout d'abord la méthode d'acquisition développée et le mode opératoire mis en œuvre pour obtenir des jeux de données image permettant la cartographie opérationnelle de zones d'intérêt. Ensuite, nous présentons les traitements effectués à l'aide de logiciels désormais abordables qui permettent le calcul de l'aérotriangulation et de la corrélation dense sur plusieurs centaines d'images. Nous présentons enfin les résultats obtenus sur des exemples d'applications en conditions réelles afin de discuter du potentiel et des limitations de cette méthode, proposée comme alternative aux solutions existantes.
\end{abstract}

Mots clés : Imagerie aérienne, Plate-forme sans pilote, Cerf-Volant, KAP, MNT, Photogrammétrie, Micmac

\begin{abstract}
This paper describes a new method using kites for very high resolution aerial image acquisition and processing. This method is proposed as an alternative to methods involving Unmanned Aerial Systems when the latter cannot be deployed in field, due to bad weather conditions or local regulations. The main issue when acquiring aerial imagery from kite being platform control, progresses undertaken to cope with this limitation and enable decimetric 3D cartography of target areas with surfaces exceeding one square kilometer are presented. The specific acquisition protocol and processing chain are described. Results obtained on two test sites demonstrate the efficiency of the method whose potential and limits are discussed.
\end{abstract}

Keywords : Aerial imagery, UAS/UAV, kite, KAP, DEM, Photogrammetry, Micmac

\section{Introduction}

Les drones ont atteint la maturité technologique qui leur permet de répondre à la plupart des besoins de l'information spatiale de très haute résolution spatiale et/ou temporelle. Colomina et Molina (2014) proposent une revue récente de leur utilisation en télédétection. Les ap- plications civiles sont nombreuses, allant de l'agriculture et de l'environnement au génie civil en passant par la surveillance et la sécurité. Elles ne demandent qu'à se développer ${ }^{1}$. Selon ces auteurs, le principal frein au développement de ce marché aujourd'hui serait en effet la

1. "Let them fly and they will create a market" 
contrainte réglementaire. Une autre limitation actuelle est l'autonomie, particulièrement en conditions très ventées.

Enfin et de manière générale, le traitement des données pose une limite à tous les travaux de calcul du relief à partir de drones (Harwin et Lucieer, 2012). Ces auteurs ont obtenu une carte de 30 millions de points sur 5 ha (3 à $5 \mathrm{~cm}$ entre deux points) en utilisant Photoscan(TM) associé à des outils spécialement développés pour l'occasion. Ils insistent sur la grande difficulté à obtenir un tel résultat avec les outils photogrammétriques actuellement disponibles.

Le cerf-volant est quant à lui utilisé de longue date comme vecteur pour porter des appareils photo. Les archéologues en sont les utilisateurs les plus connus (voir par exemple Verhoeven (2009) pour un aperçu historique). Même depuis l'avènement des drones, nombre d'entre eux restent fidèles à ce vecteur facile à manier, bon marché et sur lequel la charge utile a peu de risque d'être endommagée. Plus marginalement, le cerf-volant peut présenter un intérêt pour des applications nécessitant de limiter les perturbations sonores (Fraser et al., 1999).

Le principal problème posé par l'utilisation du cerfvolant comme vecteur est, comme remarqué entre autres par Murray et al. (2013), le manque d'agilité de la plateforme. L'obtention de jeux de données tenant un cahier des charges fixé à l'avance est délicat ou impossible. En particulier, la cartographie de grandes surfaces et/ou à très haute résolution spatiale est particulièrement problématique car elle nécessite l'assemblage de plusieurs centaines d'images alors que l'incertitude sur la position et l'altitude du cerf-volant impose une forte redondance des images.

De fait, un bon nombre de références de la littérature concerne l'exploitation de clichés individuels ou de quelques clichés sur des zones réduites, de l'ordre de quelques hectares; le maximum de surface couverte dans la littérature, de 17 hectares, correspond à une juxtaposition d'acquisitions individuelles réalisées sur plusieurs zones (Bogacki et al., 2010). Ces auteurs relèvent d'ailleurs la difficulté de couvrir de grandes surfaces lors de l'acquisition puis de gérer un grand nombre de vue stéréoscopiques lors du traitement. De plus, la technicité élevée des solutions mises en œuvre dans la littérature implique le recours à des compétences spécifiques, du matériel sophistiqué, et plusieurs opérateurs, comme illustré par exemple par Marzolff et al. (2002).

Dans ce contexte, l'objectif de ce travail est de proposer une méthode opérationnelle d'acquisition et de traitement d'imagerie aérienne basse altitude qui soit simple, robuste et transférable et qui permette la production de modèles numériques de surface et d'orthophotos de plusieurs gigapixels, donnant accès à des données spatiales 3D de résolution décimétrique sur des surfaces dépassant le kilomètre carré.

L'approche choisie est d'une part de développer un protocole qui soit à la fois léger et robuste et d'autre part, de mobiliser des chaînes photogrammétriques avancées gratuites telles que Micmac, suite open-source dévelop- pée à l'IGN (Pierrot-Deseilligny et Paparoditis, 2006).

Après une présentation de la méthode développée, nous montrons et examinons les résultats de sa mise en œuvre sur deux sites situés au nord-est de la Tunisie afin de déterminer le potentiel d'une telle méthode, d'une part vis-à-vis de la qualité des produits cartographiques obtenus et d'autre part vis-à-vis de ses possibilités opérationnelles.

\section{Matériel et Méthodes}

\subsection{Principes généraux}

\subsubsection{L'AutoKAP, technique simple et robuste}

Comme présenté en introduction, le besoin d'utiliser un cerf-volant comme plate-forme d'acquisition peut être imposé par les conditions de vent ou de réglementation. Un autre critère fort à la base de ce travail est de proposer une solution simple à mettre en œuvre, bon marché, robuste, facilement transportable et transférable. L'acquisition de type «AutoKAP» répond à ces objectifs. II s'agit d'un appareil photo suspendu à un cerf-volant («KAP» signifiant Kite Aerial Photography). Les prises de vues sont réalisées automatiquement, à intervalle régulier, sans contrôle à distance des images obtenues. Cette méthode présente l'avantage de ne nécessiter aucune intervention sur l'appareil photo, l'opérateur n'ayant qu'à manipuler la ligne du cerf-volant. Le dispositif est par ailleurs remarquablement simple puisque de nombreux appareils photo du commerce sont nativement dotés d'intervallomètre. II suffit donc de les suspendre à la ligne du cerfvolant par un dispositif rudimentaire qui permette la prise de vue au nadir.

\subsubsection{Imposition de contraintes a priori sur l'acquisition}

L'hypothèse maîtresse à la base du développement de la méthode proposée ci-après est que le vecteur (opérateur-appareil) est constant et donc que les déplacements de l'opérateur sur le terrain et de l'appareil photo sous le cerf-volant sont identiques à une translation près. L'acquisition des images souhaitées sur la zone cible est donc réalisée en parcourant le site d'étude selon un plan de déplacement déterminé à l'avance. On s'approche le plus possible de conditions où cette hypothèse est vraie en obtenant une caractérisation fine du comportement en vol des porteurs et en effectuant une série de choix sur le protocole de prise de vue selon les critères détaillés ci-après.

\subsection{Matériel utilisé}

Le matériel de prise de vue se compose d'un porteur (le cerf-volant), d'un dispositif de retenue (la ligne, ou fil), d'une plate-forme et d'une charge utile. En plus de ce matériel - détaillé ci-dessous - deux enregistreurs GPS de type QSTARZ BT1400S, choisis selon des critères de coût, de poids et d'autonomie, ont été utilisés. Le premier GPS est utilisé pour enregistrer la position de l'appareil photo, le second pour celle de l'opérateur. 


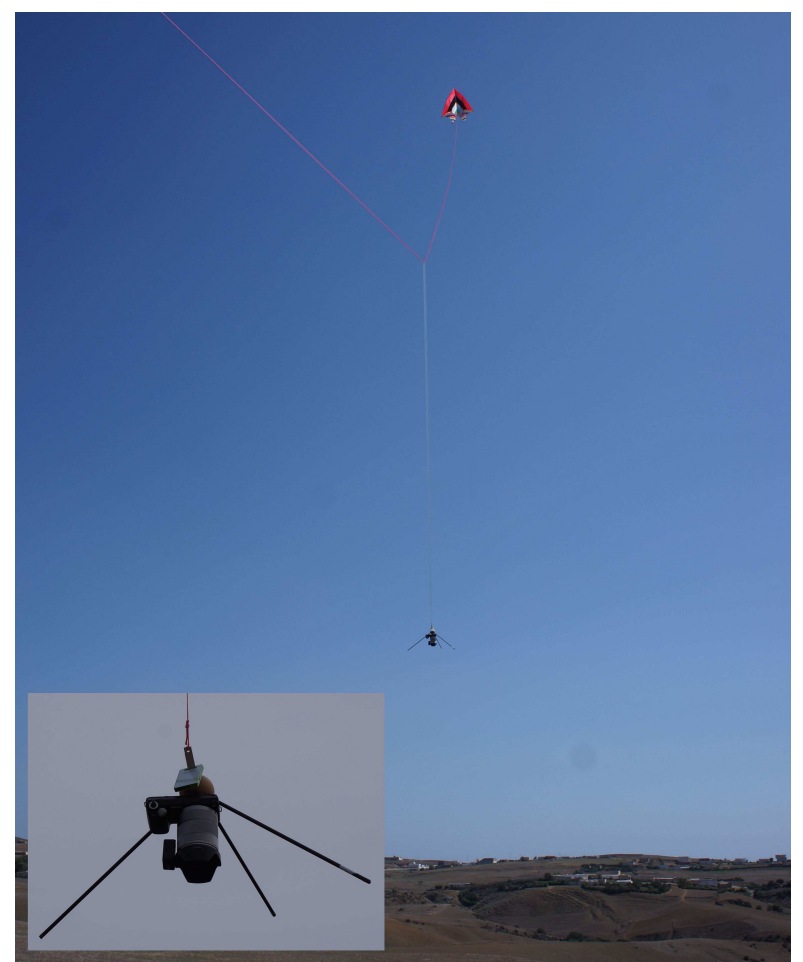

FIGURE 1: Dispositif de prise de vue avec pendule simple. En encadré, vue rapprochée de l'appareil photo muni d'un tripode. Le déclencheur automatique est fixé sur le pare soleil et l'enregistreur GPS est accroché au sommet de la plate-forme.

\subsubsection{Porteur}

II existe une très grande variété de formes de cerfvolants porteurs, à armatures (deltas, rokkakus, à cellules) ou non (flowform). Chaque forme a des caractéristiques spécifiques en terme de capacité de charge utile, de robustesse, stabilité, traction, angle de fil, facilité de réglage, de montage, de transport, etc... (voir p.e. le site de Christian Becot (http ://www.becot.info) pour plus de détails). II a été choisi de favoriser un porteur de conception simple, avec armature, sans réglages et permettant un vol avec fort angle de fil. Les premiers critères visent à disposer d'une solution sûre, simple, robuste et accessible. Enfin un vol à angle de fil fort présente plusieurs avantages parmi lesquels ceux de limiter la variabilité de l'altitude à longueur de fil donnée et d'être plus facilement manoeuvrable (traversée de végétation, zones étroites). Ces critères nous ont guidé vers les ailes de type Delta.

\subsubsection{Plate-forme}

Pour des questions de simplicité et de robustesse, il a été décidé d'utiliser un pendule simple. Le pendule a été constitué avec une grande longueur de fil de manière à minimiser les vitesses de déplacement de l'appareil lors de mouvement de balancier et ainsi minimiser les risques de flou de bougé et de filé. Un tripode a été confectionné afin de rendre possible le décollage et l'atterrissage de la plate-forme par un opérateur seul sans risque de détérioration (cf. figure 1). Un effet intéressant de l'utilisation du tripode est que, par effet girouette, sa géométrie permet de stabiliser la plate-forme en lacet. La structure centrale de la plate-forme est une languette d'aluminium qui peut être déformée de manière à régler in situ la verticale de l'appareil. Le GPS qui enregistre la position de l'appareil est glissé sur cette languette.

\subsubsection{Charge utile}

Pour l'appareil photo, le premier critère est un compromis entre prix, poids et qualités optique et géométrique. De plus les fonctions de stabilisation optique et d'autofocus doivent pouvoir être désactivées afin que la géométrie interne de prise de vue soit fixe. Cet ensemble de critères a guidé le choix vers un compact hybride à objectifs interchangeables. Au moment de l'étude, c'est le SONY NEX-5N qui présentait le meilleur rapport qualité/prix de sa catégorie et qui a été choisi. L'appareil photo est utilisé avec un objectif grand angle afin de voler le plus bas possible. Ceci permet de minimiser les éventuels problèmes liés aux prises de vue non verticales tout en facilitant le contrôle visuel du système d'acquisition une fois en vol. Le déclenchement automatique est assuré par un intervallomètre gentLED-Auto de type 05C, fixé au pare-soleil de l'objectif.

\subsubsection{Dispositif de retenue}

Le poids du fil s'ajoute à la charge utile. Un fil léger permet donc un angle de fil plus grand. La ligne choisie est du fil Dyneema de $200 \mathrm{lb}(90 \mathrm{~kg}$ ) qui pèse 0,4 $\mathrm{g} / \mathrm{m}$, soit $120 \mathrm{~g}$ pour un distance de fil de $300 \mathrm{~m}$ : le fil ne représente que $20 \%$ environ de la charge totale pour une charge utile de $500 \mathrm{~g}$. La bobine est un enrouleur avec poignée interne sur l'axe et manivelle de type boule. L'opérateur est équipé de gants de cuir, d'une grande sangle et de mousquetons (dégaines). Le fil est marqué tous les dix mètres afin de contrôler en tout temps la longueur déroulée.

\subsection{Préparation amont}

\subsubsection{Caractérisation du porteur et des appareils}

La caractérisation du comportement en vol du porteur a été réalisée en exploitant les données GPS acquises au niveau de l'appareil photo et au niveau de l'opérateur lors de premiers vols. Plusieurs vols ont été réalisés dans différentes conditions de vent et, pour chaque vol, à plusieurs longueurs de fil correspondant à différentes altitudes, afin d'étudier la variabilité de l'angle de fil pour des longueurs de fil fixe. La figure 2 présente une illustration d'une partie des mesures effectuées. L'ensemble des mesures réalisées a permis de déterminer les angles de fil moyens du cerf-volant pour différentes altitudes et vitesses de vent.

Les autonomies des différents appareils électriques sont évaluées expérimentalement. Pour l'appareil photo l'écran a été réglé à luminosité minimum et pour l'affichage des informations techniques uniquement (pas d'image) afin de minimiser la consommation électrique. Avec des conditions de vent favorables, une carte mémoire de 64Go, un intervalle de prise de vue de 5 secondes et un intervalle de 1 seconde pour l'enregistreur 


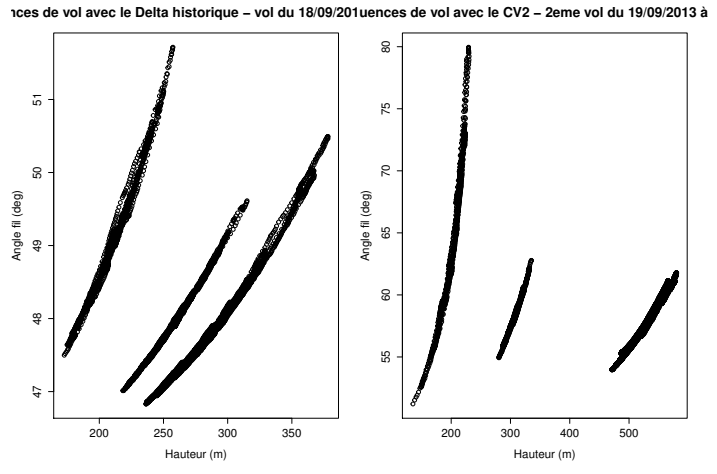

FIGURE 2: Exemple de caractéristiques mesurées pour deux ailes delta différentes. La figure de gauche présente les mesures réalisées lors d'un vol avec une aile delta de $4 \mathrm{~m}^{2}$ à 3 longueurs de fil différentes. La figure de droite présente les mesures réalisées lors d'un vol avec une aile delta de $10 \mathrm{~m}^{2}$, là encore avec 3 longueurs de fil différentes.

GPS, c'est dans notre cas la batterie de l'appareil photo qui est le facteur limitant. L'autonomie de prise de vues avec ces réglages est de plus de $3 \mathrm{~h} 30 \mathrm{~min}$, soit plus de 2500 clichés.

\subsection{2. Étude et documentation du site}

Si possible, un repérage est effectué sur le terrain. Une carte du site est préparée et imprimée sur des feuilles A4. Faute de mieux, les images Google Earth couvrant le site sont extraites, assemblées, et imprimées à une échelle adaptée au chantier. Lors de l'acquisition, une règle de réduction en éventail est utilisée afin mesurer directement les distances terrain sur les plans de repérage. Ceux-ci sont glissés dans des pochettes transparentes utilisées pour la prise de note, notamment le report des emprises au sol des images, réalisé en contrôle dès l'atterrissage.

\subsubsection{Détermination des paramètres de vol - appareil photo}

Les paramètres de vol sont déterminés en fonction de la zone cible et de la résolution souhaitée. L'application du théorème de Thalès à la géométrie projective globale de prise de vue donne la formule suivante:

$$
S_{p i x} / f=G S D / h \text {, d'où } G S D=h * S_{p i x} / f,
$$

avec $S_{p i x}$ la taille physique du pixel sur le capteur, $f$ la distance focale réelle, $G S D$ la taille du pixel au sol et $h$ la hauteur de prise de vue. Pour un appareil photo donné et une résolution au sol souhaitée, le rapport altitude/focale et donc figé.

Un autre paramètre important est le taux de recouvrement, pour lequel deux critères principaux interviennent. D'une part les images doivent être acquises avec des points de vue peu différents afin de maximiser la réussite des algorithmes de détection et d'appariement automatique de points utilisés (algorithme SIFT de Lowe
(1999)). D'autre part, la forte redondance d'images permet à la fois une meilleure restitution du relief par corrélation multiple et sécurise la couverture stéréoscopique du chantier. Un taux de recouvrement longitudinal de $90 \%$ et un taux latéral supérieur à $30 \%$ ont donc été visés.

Finalement, le taux de recouvrement, l'altitude, et la vitesse de marche de l'opérateur imposent une cadence de déclenchement maximale.

\subsubsection{Détermination des paramètres de vol - porteur}

L'altitude de l'appareil un fois fixée, les paramètres de vol au niveau du porteur sont déterminés. Si le terrain le permet, on choisit le porteur ayant le plus grand angle de fil. Ceci limite l'incertitude sur les altitudes et donc les variations d'échelle / de résolution au sol d'une image à l'autre. Dans certains cas (zones inaccessibles à pied par exemple), il peut être intéressant de choisir un porteur avec un angle de fil faible. On obtient ainsi une plus grande distance entre opérateur et appareil avec une même longueur de fil. Cette solution n'est cependant pas à privilégier du fait que l'incertitude sur l'altitude est plus importante pour de faibles angles de fil.

Plusieurs dizaines de mètres de fil minimum sont déroulés entre le cerf-volant et l'appareil de manière à ce que les mouvements du cerf-volant soient amortis au niveau de l'appareil. En fonction des caractéristiques en vol du cerf-volant et pour des prises de vues à haute résolution imposant que l'appareil soit à très basse altitude, il peut être intéressant de mettre le cerf-volant significativement plus haut que l'appareil (cf figure 2 à droite : les variations d'angle sont moins importantes à plus haute altitude). L'altitude cible du cerf-volant détermine la longueur de fil à dérouler entre l'appareil et le cerf-volant.

\subsection{5. Établissement du plan d'acquisition}

Le plan de vol - en fait un plan de marche, comme exposé plus haut - est déterminé en fonction de la zone cible à couvrir. II est constitué d'allers-retours avec une inter-distance correspondant au recouvrement latéral visé. Les allers-retours sont réalisés perpendiculairement au vent et en descendant le vent. Cette technique permet de minimiser et de gérer les variations de tension du fil et donc de favoriser la stabilité du vecteur (opérateurappareil).

Finalement les deux altitudes (appareil et cerf-volant) et l'angle de fil permettent de déterminer la longueur de fil à dérouler entre le cerf-volant et l'appareil photo tout d'abord, puis entre l'appareil photo et l'opérateur. La distance entre opérateur et point au sol situé à la verticale de l'appareil (soit le centre de l'image pour des visées parfaitement verticales) est calculée, ce qui fixe le décalage horizontal entre parcours à pied et plan de vol.

\subsection{Acquisition des images}

Si possible, la sortie est programmée en fonction des conditions météorologiques facilitant l'acquisition : luminosité suffisante et homogène ${ }^{2}$, vent régulier, direction

2. pour les acquisitions à base altitude, la présence d'un voile nuageux n'est pas génante et peut même être un atout 
du vent perpendiculaire à la pente dominante du site par exemple.

\subsubsection{Mise en place du matériel}

À l'arrivée sur le site le le cerf-volant est envoyé dès que possible. Le comportement du cerf-volant est observé pendant la préparation du reste du matériel. La direction du vent détermine le point de décollage, à la fois au vent de la zone à imager et sous le vent d'une zone dégagée d'obstacles (forêts/ bâtiments). La force du vent au sol est évaluée en observant le paysage suivant l'échelle Beaufort terrestre. Le cerf-volant est choisi en fonction de cette force, en tenant compte du fait que le vent en altitude est plus important qu'au sol. Le cerfvolant est monté, attaché à la ligne et la longueur de fil déterminée à l'avance est dévidée. Le cerf-volant est ancré et obligatoirement maintenu sous surveillance constante. La plate-forme et la charge utile sont préparées, les deux GPS enregistreurs sont démarrés. L'appareil photo est réglé en mode sport et la mise au point bloquée sur l'infini. La plate-forme est réglée de manière à ce que l'appareil en place ait une visée verticale. Une fois l'ensemble de la charge utile prêt, on effectue une photo de vérification afin de sécuriser l'acquisition réalisée par la suite en aveugle.

\subsubsection{Réalisation de l'acquisition}

Une fois tous les contrôles effectués, l'intervallomètre peut être démarré et la deuxième portion de longueur de fil dévidée afin d'amener l'appareil à l'altitude cible. L'heure de démarrage est notée afin d'évaluer l'heure de fin d'acquisition en fonction de l'autonomie déterminée. L'appareil stabilisé à l'altitude visée, le parcours à pied prévu est réalisé. En fin d'acquisition, le fil de retenue est à nouveau ancré. Pour descendre le porteur on utilise une poulie pour amener le fil au sol en marchant vers le cerf-volant. Si les conditions de terrain l'imposent, des allers-retours sont réalisés, un noeud étant réalisé au niveau de la poulie pour que l'enroulement du fil soit réalisé hors tension.

\subsubsection{Synchro GPS - Vérification de la couverture de la zone cible}

Afin d'associer les coordonnées GPS aux images, il est nécessaire de synchroniser les horloges de l'appareil photo et du GPS. Pour ce faire on prend en photo - avec le même protocole (intervalle) qu'en vol - un écran qui affiche l'heure GPS en temps réel.

Les images acquises en vol sont examinées et les contours d'un sous-ensemble de ces images sont tracés à main levée sur les plans de repérage afin de vérifier la bonne couverture stéréo de la zone cible avec des images de qualité.

\subsection{Traitement des données}

\subsubsection{Pré-traitements : synchronisation GPS, prépara- tion de fichiers}

Les photos de synchronisation GPS sont exploitées pour calculer la moyenne de la différence entre temps
GPS et horodatage des photos (tag exif DateTimeOriginal) et ainsi le décalage temporel entre horloge de l'appareil photo et temps GPS.

Par ailleurs, les images sont triées afin de ne conserver que la partie exploitable. L'intervallomètre étant démarré dès le décollage de l'appareil photo, le jeu de données contient plusieurs images superflues, notamment celles de décollage et d'atterrissage.

Enfin, le dépouillement des enregistreurs GPS permet de créer un fichier contenant les coordonnées de l'appareil au moment de chaque prise de vue ainsi que la position de l'opérateur au même instant. Ces données sont utilisées à la fois pour caractériser le vol du porteur mais aussi pour accéder à un géoréférencement absolu du chantier dans le cas où on ne dispose pas de points d'appui au sol.

\subsubsection{Traitements photogrammétriques}

Ces traitements sont effectués avec la chaîne opensource Micmac (Pierrot-Deseilligny et Paparoditis, 2006), distribuée sous la licence ( CeCILL-B ) permettant une utilisation libre en échange d'une obligation de citation. La chaîne minimale contient 4 étapes : 1) calcul automatique et appariement de points de liaisons (Lowe (1999) 2) calcul de la géométrie interne (focale, centre de projection, distorsion) et externe (position et angle de visée de la caméra) 3) corrélation dense : appariement des images par la mise en correspondance à l'échelle du pixel pour le calcul du relief et des orthophotos et 4) assemblage des orthophotos et égalisation radiométrique.

Il est recommandé d'acquérir un jeu de données spécifique pour la calibration de la géométrie interne (distorsion de la lentille), en utilisant l'appareil dans les mêmes conditions que lors de l'acquisition en vol (la bague de mise au point aura pu être maintenue en position fixe par un ruban de bande adhésive). La scène idéale pour la calibration est une scène $3 \mathrm{D}$ très texturée, qui sera prise en visée convergente (le centre de l'image visant toujours le même point) avec différents points de vue et en tournant l'appareil.

La suite Micmac est constituée d'une succession de programmes appelés en ligne de commande, par exemple pour les 4 étapes décrites ci-dessus : Tapioca, Tapas, Malt et Tawny. D'autres commandes permettant la bascule du chantier de la géométrie image vers la géométrie terrain ont été utilisées. II est possible d'exploiter les observations terrain et image de points de contrôle au sol, les observations des positions de prise de vue, ou encore d'effectuer une compensation de ces deux types de mesures en plus des contraintes imposées par l'appariement des points de liaison calculés automatiquement.

\section{Résultats}

La méthode présentée ici a été développée et testée sur deux sites situés au Nord-Est de la Tunisie sur la péninsule du Cap Bon, au sein du bassin versant du barrage sur l'oued Lebna. Un travail préparatoire permettant d'affiner la méthode et de valider son potentiel a été 
réalisé sur le site de Kamech (ORE OMERE). Un test opérationnel en vrai grandeur a ensuite été réalisé sur le site de Fortuna.

\subsection{Développement et validation de la méthode ; pre- mières applications sur le site de Kamech}

\subsubsection{Site de Kamech, ORE OMERE}

Le site de Kamech est un bassin versant expérimental de 2,63 $\mathrm{km}^{2}$ décrit en détail par Mekki et al. (2006) et Raclot et Albergel (2006). Son exutoire est un lac de retenue collinaire suivi depuis 1994 dans le cadre d'une convention d'application entre la Direction de la Conservation des Eaux et du Sol du Ministère de l'Agriculture Tunisien (DG ACTA/CES, Tunisie) et l'Institut de Recherche pour le Développement (IRD, France). II est aussi depuis 2002 l'un des deux sites de l'Observatoire de Recherche en Environnement OMERE ${ }^{3}$ (Observatoire Méditerranéen de l'Environnement Rural et de l'Eau), cogéré en Tunisie par l'IRD / UMR LISAH, I'INRGREF et I'INAT. Du point de vue de la topographie, les altitudes sur le bassin versant de Kamech varient entre 80 et 200 $\mathrm{m}$, avec des pentes dépassant localement les $100 \%$.

\subsubsection{Données de terrain disponibles}

Pour le site de Kamech, des données existantes de calage et de validation ont pu être exploitées. II s'agit de données récoltées lors d'une opération de bathymétrie réalisée du 24 au 27 juin 2013 avec un GPS différentiel RTK de type TOPCON-GR3. Les spécifications constructeur donnent une exactitude de $1 \mathrm{~cm}$ en $X Y$ et $1,5 \mathrm{~cm}$ en $Z$ avec cet appareil. Le nuage de points 3D mesuré contient des points émergés dont quelques uns repérables dans les images. 8 points situés autour du lac ont été utilisés comme points de calage et 469 autres points émergés autour du lac comme points de validation. Le restant des points (points immergés et points en dehors de la zone couverte par les points de contrôle) n'a pas été utilisé.

\subsubsection{Acquisitions réalisées}

Lors d'une session de trois jours du 17 au 19 septembre 2013, 4 vols ont été réalisés sur le site de Kamech et plus de 3000 images ont été acquises sur un total d'une dizaine d'heures de vol. Lors de chacun de ces 4 vols, les positions de l'appareil et de l'opérateur ont été enregistrées chaque seconde par les deux enregistreurs GPS. Les vols ont été réalisés avec différents porteurs et à différentes altitudes. L'objectif était d'une part de caractériser les porteurs mais aussi de réaliser une première couverture du bassin versant. La zone cible prévue a une surface de plus de $2 \mathrm{~km}^{2}$ et correspond à la quasi-totalité du bassin versant. Une petite zone située tout à fait à l'amont n'a pas été visée du fait de la présence d'une ligne électrique. Lors de cette session, le dernier vol, de $3 \mathrm{~h}$, a permis à lui seul la couverture de $80 \%$ de la surface ciblée.

3. http ://www.obs-omere.org

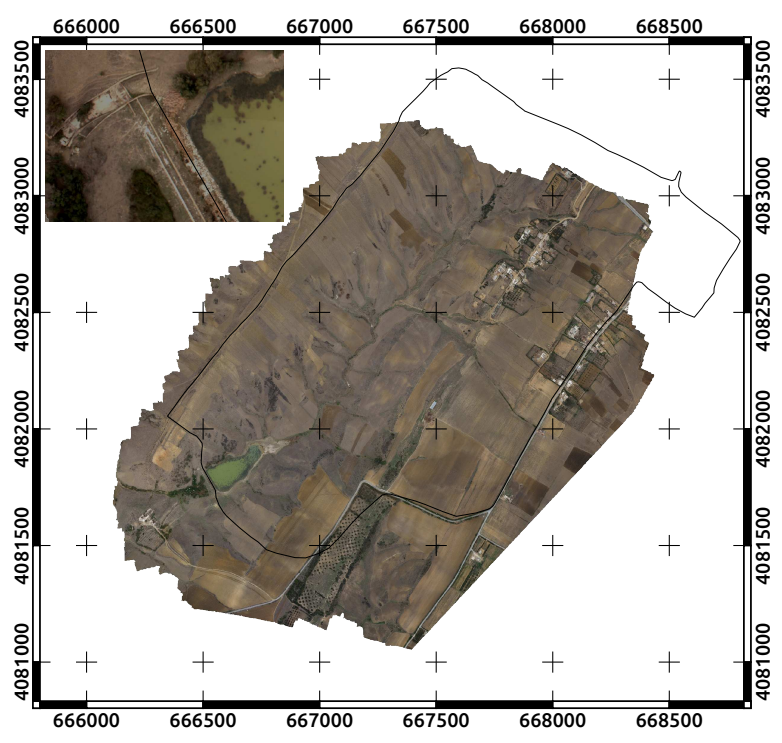

FIGURE 3: Kamech : orthophoto à $10 \mathrm{~cm}$ produite et limites du bassin versant (projection EPSG 22332 ; détail : déversoir).

\subsubsection{Produits obtenus et évaluation de leur qualité}

Le calcul du modèle numérique de surface à $20 \mathrm{~cm}$ et de l'orthophoto à $10 \mathrm{~cm}$ couvrant la zone de plus de $2 \mathrm{~km}^{2}$ a été réalisé à partir de 752 images (figures 3 et 4). La chaîne de traitements décrite plus haut a été exécutée sur un ordinateur équipé d'un processeur Intel Core i7-3840QM à 2,8 GHz (8 coeurs) et de 32 Go de mémoire vive. Le calcul et l'appariement automatique de points remarquables dans les images (Tapioca) a représenté un peu moins de $6 \mathrm{~h}$ de calcul. La mise en place du bloc (Tapas) a nécessité $12 \mathrm{~h}$, cette étape étant aussi la plus consommatrice en mémoire vive. Enfin le calcul du modèle numérique de surface et l'orthorectification des images a été effectué en un peu moins de $15 \mathrm{~h}$. La suite micmac détermine automatiquement une résolution maximale de l'orthophoto et du modèle numérique de surface ainsi que la précision en Z. Sur ce chantier, la précision planimétrique et altimétrique sont de $20 \mathrm{~cm}$ pour le modèle numérique de surface, l'orthophoto étant restituée à $10 \mathrm{~cm}$.

L'erreur de restitution altimétrique est estimée par différence entre la valeur de l'altitude restituée par photogrammétrie et la mesure effectuée par GPS différentiel RTK. L'erreur médiane observée et de $9 \mathrm{~cm}$ et l'écarttype de l'erreur de $37 \mathrm{~cm}$ (table 1 et figure 5). L'étalement de la distribution de l'erreur pour des valeurs supérieures à $1 \mathrm{~m}$ s'explique essentiellement par la présence de végétation arbustive en bordure du lac.

\subsection{Validation opérationnelle - site de Fortuna}

\subsubsection{Site de Fortuna, bassin versant d'Arrwiguet}

Faisant partie des moyennes montagnes de la dorsale tunisienne et du Cap Bon, le bassin versant Arrwiguet est représentatif d'un milieu contraignant. La topographie est marquée par une monotonie frappante. La région du bassin versant Arrwiguet se situe sur le flanc 


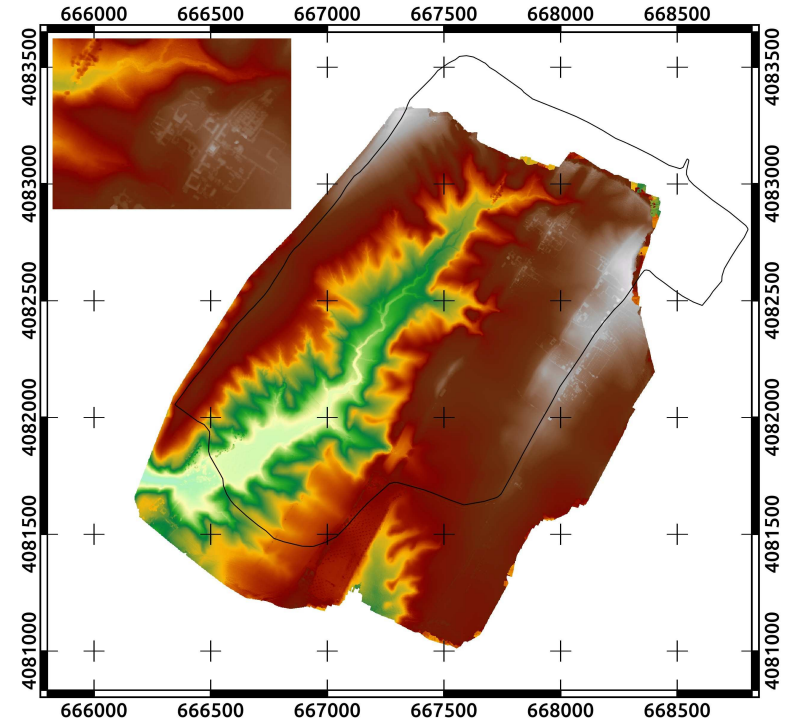

FIGURE 4: Kamech : modèle numérique de terrain à $20 \mathrm{~cm}$ (résolution planimétrique et altimétrique) et limites du bassin versant (projection EPSG 22332 ; détail : habitations).

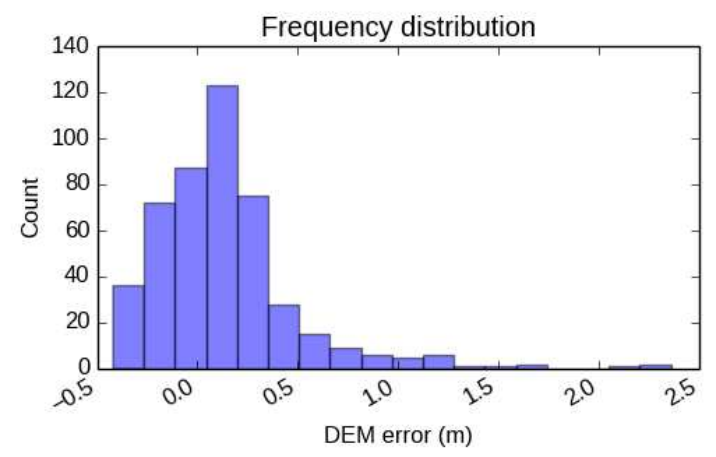

FIGURE 5: Kamech : distribution de l'erreur d'estimation de l'altitude

\begin{tabular}{|c|c|}
\hline Moyenne & $+13 \mathrm{~cm}$ \\
\hline Médiane & $+9 \mathrm{~cm}$ \\
\hline Écart-type & $37 \mathrm{~cm}$ \\
\hline Intervalle à $90 \%$ & {$[-29 \mathrm{~cm} ;+81 \mathrm{~cm}]$} \\
\hline Nombre de points & 469 \\
\hline
\end{tabular}

TABLE 1: Kamech : statistique de l'erreur oriental de l'anticlinal du djebel Sidi Abderrahmen. Le bassin est marqué par une structuration particulière des séries allant du Lutétien supérieur-Priabonien au Langhien supérieur-Serravallien. Le point culminant se situe à 450 mètres, le point le plus bas est à 70 mètres, donnant une dénivelée de 380 mètres. Son exutoire est le lac collinaire Arrwiguet. Le site où l'acquisition a été réalisée se situe entre les micro-bassins numérotés 1 et 8 sur la figure 3 de El Maaoui et al. (2012).

\subsubsection{Cahier des charges}

Sur le site de Fortuna, l'objectif était d'obtenir, sur une zone d'une vingtaine d'hectares comprenant cinq ravines (dont la ravine 8 décrite dans El Maaoui et al. (2012)), une ortophoto et un modèle numérique de terrain d'une précision en $Z$ d'une dizaine de centimètres. Afin d'assurer l'obtention de cette précision, il a été décidé d'effectuer une acquisition avec une résolution au sol d'environ $5 \mathrm{~cm}$. Avec le Sony NEX-5N équipé d'un objectif de focale $18 \mathrm{~mm}$, cela correspond à une altitude de $190 \mathrm{~m}$. En fonction de cette altitude et des données expérimentales de vol recueillies en amont, les plages de variation des angles de fil des deux cerf-volants ont été déterminées. Pour le delta de $4 \mathrm{~m}^{2}$, les angles de fil sont compris entre 46 et 50 degrés; pour le delta de 10 $\mathrm{m}^{2}$, les angles sont compris entre 54 et 62 degrés. Ces angles correspondent à des longueurs de fil entre l'opérateur et l'appareil de $250 \mathrm{~m}$ (hauteurs comprises entre $180 \mathrm{~m}$ et $192 \mathrm{~m}$ ) pour le delta de $4 \mathrm{~m}^{2}$ et de $230 \mathrm{~m}$ (hauteurs comprises approximativement entre $185 \mathrm{~m}$ et 200 m) pour le delta de $10 \mathrm{~m}^{2}$.

Pour assurer un recouvrement longitudinal de $90 \%$ avec une vitesse de marche approximative de $1 \mathrm{~m} / \mathrm{s}$, un intervalle de prise de vue d'une quinzaine de secondes maximum doit être utilisé.

\subsubsection{Réalisation du plan de vol}

Sur une fenêtre temporelle imposée d'une semaine, le jour de l'acquisition sur Fortuna a été choisi en fonction des prévisions météorologiques afin de favoriser une journée sans nuages et avec un vent modéré (force 3 à 4 beaufort). Le jour de l'acquisition, les conditions de vent ont amené à choisir le cerf-volant de $10 \mathrm{~m}^{2}$. Vue la relativement faible extension spatiale du site, la résolution cible et l'autonomie de prise de vue, il a été décidé le jour de l'acquisition d'effectuer le vol en deux temps, avec un premier passage à altitude (et donc résolution) approximativement moitié en déroulant $110 \mathrm{~m}$ de fil et un deuxième passage à la résolution cible avec $230 \mathrm{~m}$ de fil. Afin de disposer de données supplémentaire de validation du protocole, une courte séquence de vol avec $180 \mathrm{~m}$ de fil a été réalisée entre les deux séquences. Ces longueurs sont déroulées précisément grâce aux graduations réalisées sur le fil. De plus, afin de garantir le recouvrement longitudinal de $90 \%$ pour l'acquisition à mi-hauteur, l'intervallomètre a été réglé sur $5 \mathrm{~s}$.

Pour la réalisation de l'acquisition, $4 \mathrm{~h}$ se sont écoulées entre arrivée sur site, vol avec acquisition et retour au sol du matériel. Ensuite, un peu plus d'une heure et 


\begin{tabular}{|c|c|c|c|}
\hline \multicolumn{2}{|c|}{ Distance directe $(\mathrm{m})$} & \multicolumn{2}{c|}{ Altitude $(\mathrm{m})$} \\
\hline Prévue & Observées & Prévues & Observées \\
\hline 110 & {$[105 ; 114]$} & {$[89 ; 97]$} & {$[77 ; 106]$} \\
\hline 180 & {$[176 ; 181]$} & {$[146 ; 159]$} & {$[138 ; 163]$} \\
\hline 230 & {$[220 ; 234]$} & {$[186 ; 203]$} & {$\left[106^{*} ; 212\right]$} \\
\hline
\end{tabular}

TABLE 2: Comparaison entre valeurs prévues et observées pour la distance directe opérateur/appareil et l'altitude. ${ }^{*}$ Cette valeur aberrante est due à une diminution passagère du vent

demie ont été nécessaires pour effectuer le dépouillement en direct des images et le rangement du matériel. Plus de 1500 images ont été acquises; une fois l'acquisition réalisée, les emprises au sol d'une sélection des images ont été reportées à main levée sur les plans de repérage de manière à vérifier la couverture complète et en stéréo des 5 ravines cibles.

\subsubsection{Validation a posteriori du plan de vol}

Les traces GPS de l'opérateur et de l'appareil photo ont été dépouillées afin de vérifier le respect des paramètres de vol fixés et ainsi valider le caractère opérationnel de la méthode proposée.

On a vérifié tout d'abord l'hypothèse forte sur laquelle repose la méthode : le fait que l'imposition d'une longueur de fil au sol impose une position stable de l'appareil et un vecteur (opérateur, appareil) approximativement constant. Cette hypothèse est vérifiée en comparant la longueur de fil donnée et estimée à partir des marques avec la distance appareil/opérateur estimée par GPS ainsi que les altitudes prévues et réalisées (table 2).

On constate que l'erreur sur la distance directe est inférieure à $5 \%$, ce qui valide d'une part l'intérêt du marquage du fil mais surtout le fait que le fil reste bien tendu (distance constante). On constate par ailleurs que l'erreur sur l'altitude (et donc sur la résolution) est de l'ordre de $10 \%$.

Des premiers traitements ont été réalisés sur le sousensemble de 77 images prises à la plus basse altitude et couvrant les 3 premières ravines. On obtient une orthophoto à $2,5 \mathrm{~cm}$ et un modèle numérique de terrain à 5 $\mathrm{cm}$ (figures 6 et 7 ).

\section{Discussion}

\subsection{Qualité des produits obtenus}

La précision de positionnement n'a pas été évaluée quantitativement du fait du faible nombre de points de contrôle disponibles. II est cependant raisonnable de considérer que - tant que la taille du pixel est supérieure à la précision de positionnement de ces points - la précision de positionnement est de l'ordre de grandeur du pixel au sol. Par ailleurs un test a été effectué en utilisant seulement les estimations GPS des positions de l'appareil photo et en examinant qualitativement les décalages : la précision obtenue est de l'ordre d'une dizaine de mètres.

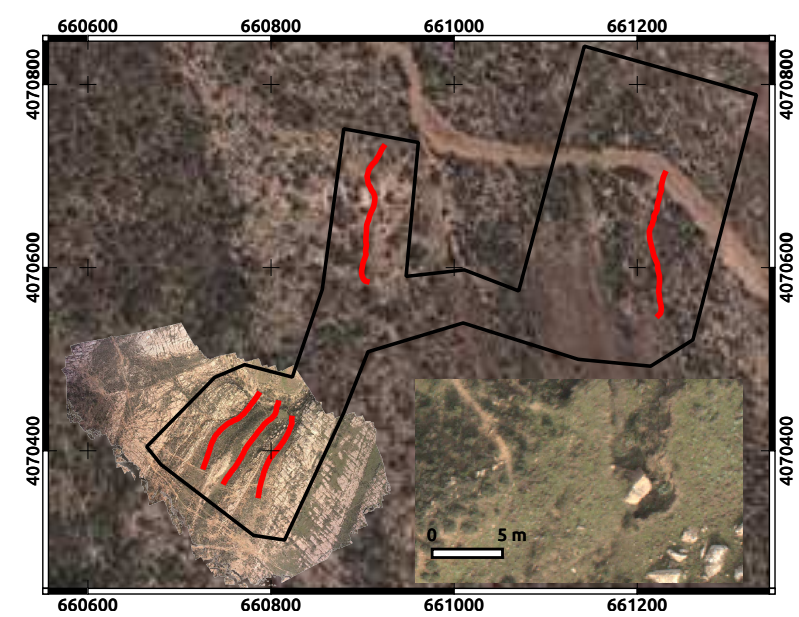

FIGURE 6: Fortuna : zone cible, ravins à imager et orthophoto réalisée à partir d'un premier sous-ensemble de 77 images (projection EPSG 22332)

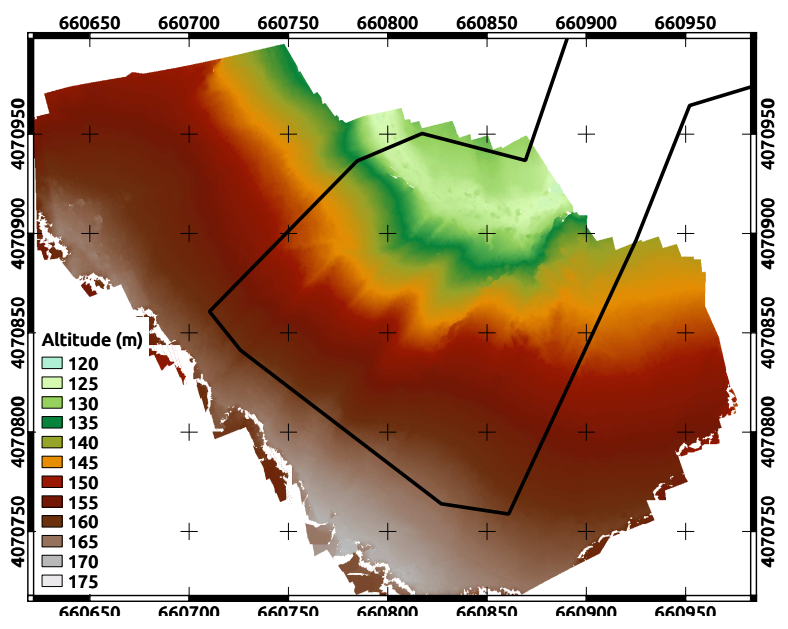

FIGURE 7: Fortuna : modèle numérique de terrain à $5 \mathrm{~cm}$ (résolution altimétrique : $2,25 \mathrm{~cm}$ ) réalisé à partir du sous-ensemble de 77 images (projection EPSG 22332) 


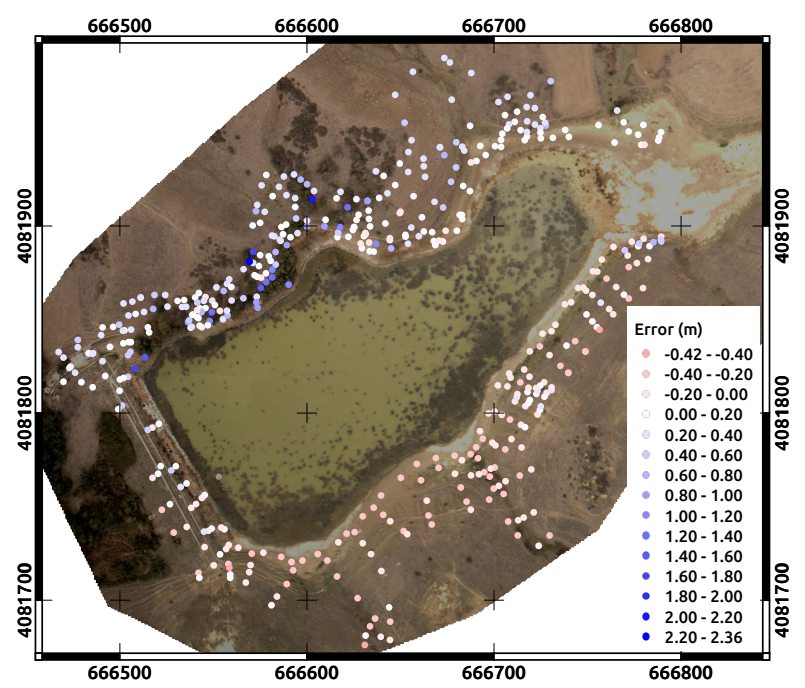

FIGURE 8: Kamech : distribution spatiale de l'erreur d'estimation de l'altitude

En ce qui concerne la précision de restitution en Z, comme montré ci-dessus elle est de l'ordre de la taille du pixel au sol. L'étude de la carte des erreurs (figure 8) permet d'émettre plusieurs hypothèses pouvant expliquer l'erreur constatée.

D'une part, on observe des erreurs de grande magnitude (supérieures à $1 \mathrm{~m}$ ) sur les zones couvertes de végétation. Ces erreurs sont dues au fait que l'on compare un modèle numérique de surface (tiré des images) avec des points relevés au niveau du sol ; ces deux surfaces sont différentes sur les zones couvertes de végétation (essentiellement en rive droite mais aussi sur un petit bosquet en bordure de plan d'eau à l'amont en rive gauche). Un traitement particulier, s'appuyant sur les informations présentes dans les images par exemple, pourrait être mis en œuvre afin de distinguer les points de sol des points de sur-sol et ainsi post-traiter le modèle numérique de surface pour obtenir un modèle numérique de terrain.

D'autre part, on observe une erreur systématique de grande longueur d'onde spatiale, les altitudes des points situés en rive droite étant en moyenne sur-estimées alors que les altitudes des points situés en rive gauche sont en moyenne sous-estimées. Cette erreur peut être la conséquence d'un mauvais calage absolu du chantier, résultant en un léger basculement du modèle numérique de surface. Ce mauvais calage est probablement lié au fait que 3 points situés sur la rive gauche sont des piquets. La résolution de prise de vue ne permettant pas de faire la différence entre haut et bas du piquet, une incertitude de l'ordre de grandeur de la hauteur de ces piquets a été spécifiée pour l'altitude de ces points de contrôle. II est supposé que le manque de contrainte sur ces points a conduit au basculement constaté.

\subsection{Caractère opérationnel de la méthode}

\subsubsection{Potentiel}

L'application de la méthode proposée sur un deuxième terrain, moins connu, l'acquisition ayant été réalisée sur une seule journée, démontre que la méthode proposée est opérationnelle et reproductible. La méthode est applicable en conditions de vent modéré à fort, conditions qui sont défavorables a priori au déploiement de drones de type multirotor. Par ailleurs cette méthode peut être mobilisée lorsque des contraintes réglementaires telles que les autorisations de vol ou la retenue du matériel en douane pour des acquisition à l'étranger empêchent l'utilisation d'autres outils.

L'acquisition réalisée sur le site de Kamech a démontré la faisabilité de la couverture décimétrique d'une zone de plus de $2 \mathrm{~km}^{2}$, en utilisant 752 images. Dans de bonnes conditions d'acquisition (terrain et météo) et moyennant une préparation suffisante du vol (repérage sur image satellite haute résolution, caractérisation du matériel), il est possible en un peu plus de trois heures d'acquérir plus de 2000 images. Avec le même appareil (16 Mpixels), un recouvrement longitudinal de $90 \%$ et latéral de $50 \%$ et des moyens de calcul adaptés, le potentiel d'obtention d'une orthoimage associée à une modèle 3D est supérieur au gigapixel, ce qui représente une couverture meilleure que décimétrique de zones de plusieurs kilomètres carrés.

\subsubsection{Limitations}

La méthode développée présente des limitations de différents ordres, tant au niveau de l'acquisition que du traitement.

Les limitations principales se situent probablement au niveau de l'acquisition : il est en effet nécessaire de pouvoir effectuer le parcours à pied afin d'obtenir un couverture stéréo sans lacune de la zone cible. Ce parcours peut s'avérer impossible dans les zones de végétation dense ou dans les zones inaccessibles à pied. Comme évoqué plus haut, il est possible pour gérer ce type de situation de jouer sur le choix du cerf-volant en privilégiant des porteurs à angle de fil plus faibles permettant des acquisitions déportées, mais au prix d'une fiabilité moindre du protocole.

Au niveau du traitement, la réalisation de calculs à partir de gros jeux de données nécessite, avec les outils disponibles actuellement, d'importantes ressources, tout particulièrement pour l'étape de calcul de la géométrie de prise de vue. Cette étape correspond en effet à la résolution de systèmes impliquant la mise en mémoire vive de toutes les paires de points de liaison (points SIFT) repérées sur l'ensemble du chantier. La taille mémoire nécessaire est donc en $O\left(n^{2}\right)$ du nombre d'images utilisées. Sur la chaîne Micmac, des développements sont en cours afin de décimer de manière optimale les points SIFT en éliminant les points surnuméraires (trop denses) ou de moindre qualité (points non multiples). Ces développements augmenteront considérablement la taille des chantiers réalisables. 


\subsection{Améliorations envisagées}

\subsubsection{Au niveau de l'acquisition}

La mise en œuvre de moyens de télétransmission radio "classiques" (radiocommande, retour vidéo) n'est pas envisagée afin de conserver le caractère simple, robuste et "passe-partout" de la méthode ainsi que son faible coût. Cependant la mise en œuvre d'appareils disposant du Wi-Fi pourrait permettre d'améliorer le contrôle en direct de l'acquisition. De même, l'utilisation d'une plateforme intelligente, s'inspirant des principes proposés par Murray et al. (2013) est à étudier. Ceci dit, les améliorations envisagées à court terme au niveau de l'acquisition concernent surtout le segment sol.

D'une part il est possible d'améliorer et de sécuriser l'acquisition en utilisant une tablette ou un smartphone pour tracer en temps réel le parcours réalisé à pied sur un fond de carte afin d'effectuer un meilleur contrôle du plan d'acquisition.

D'autre part, vu le déséquilibre entre échantillonnage le long des lignes de vol et perpendiculairement aux lignes de vol, il pourrait être intéressant de réaliser une ou deux lignes de vol perpendiculairement aux autres. Ceci permettrait de mieux contraindre la géométrie d'acquisition tant par les points de liaison que par compensation des données GPS. Par ailleurs, l'usage d'un module GPS permettant la mesure différentielle en temps réel permettrait, sans acquisition de points de contrôle au sol, d'améliorer significativement la précision absolue de positionnement.

Enfin, il est clair que l'utilisation de points de contrôle au sol dédiés, disposés avant le vol de manière régulière sur la zone cible apporterait une amélioration très importante du positionnement en planimétrie, mais aussi en altitude (cf. analyse de l'erreur montrée sur la figure 8). Cette solution implique cependant une logistique terrain plus lourde et plus coûteuse.

\subsubsection{Au niveau du traitement}

Au niveau du traitement, des améliorations peuvent être apportées par le développement d'outils automatisés dédiés au traitement d'images acquises par ce type de plates-formes. L'idée maîtresse de la méthode résidant à la fois dans l'utilisation de contraintes a priori sur le protocole et dans la redondance des images afin de s'assurer de réaliser une couverture stéréo complète d'une zone cible, les jeux d'images obtenus sont souvent sousoptimaux. Même si des premiers outils ont été testés pour le pré-traitement des images, il reste de nombreux développements à effectuer à cette étape.

D'une part, malgré l'utilisation de porteurs particulièrement stables, d'un pendule de grande longueur et de réglages spécifiques de l'appareil, un nombre non négligeable d'images floues subsiste. Des algorithmes tels que celui de Hassen et al. (2013) ou celui Crete et al. $(2007)^{4}$ ont été testés mais la question de la sélection automatique des images à utiliser en fonction de ces indices reste non résolue.

\footnotetext{
4. implémenté par Do Quoc Bao
}

D'autre part, et en particulier pour certaines configurations de terrain (ou lors d'acquisitions réalisées en plusieurs fois), il est possible que plusieurs sous-ensembles d'images de caractéristiques très différentes permettent la couverture complète de la zone cible. Il serait intéressant de développer des outils pour déterminer ces différents sous-ensembles d'images afin d'optimiser et d'automatiser ces parties de la chaîne de traitement.

\section{Conclusion}

Le travail proposé ici est parti du constat du besoin de solutions alternatives aux drones multirotor pour la cartographie $3 \mathrm{D}$ à résolution décimétrique sur des domaines de l'ordre du kilomètre. En effet, sous certaines conditions, ces plates-formes ne peuvent pas être mises en œuvre :

- contraintes réglementaires

- contraintes météorologiques (zones venteuses)

- coût d'acquisition

- coût indirects liés à la technicité nécessaire

Une méthodologie d'acquisition et de traitement de photographies aériennes par cerf-volant permettant l'obtention de telles données a donc été développée et décrite.

La méthode a été développée, mise en œuvre et testée sur un premier site ; le caractère reproductible et opérationnel de la méthode a été vérifié par sa mise en œuvre sur un deuxième site indépendant. Le test réalisé sur le second site a permis de démontrer le potentiel opérationnel de la méthode en vérifiant l'adéquation entre cahier des charges de l'acquisition et réalisation effective. Les produits obtenus par cette méthode ont été évalués et il a été vérifié que la méthode proposée permet d'obtenir une couverture décimétrique complète des zones cibles et une estimation du relief avec une précision altimétrique décimétrique (de l'ordre de grandeur de la taille du pixel des images acquises sous cerf-volant).

L'examen des erreurs d'estimation de l'altitude montre que si une part peut être imputée à la différence entre modèle de surface et modèle de terrain, des erreurs de calage absolu du bloc d'images subsistent. Ces erreurs peuvent être réduites, en particulier par l'usage d'un meilleur jeu de points de contrôle au sol (type de points, quantité, répartition) si un calage absolu fin des altitudes est nécessaire. L'usage de modules GPS permettant la correction différentielle en temps réel pourrait aussi permettre d'améliorer le positionnement absolu et donc l'estimation d'altitudes absolues.

Les voies d'amélioration dégagées se portent sur l'usage de technologies légères pour l'appui à l'acquisition (suivi temps réel du parcours de l'opérateur sur tablette ou smartphone, voire utilisation d'appareils photo numériques équipés de $\mathrm{Wi}-\mathrm{Fi}$ ) mais une complexification de la charge utile rendant le système fragile ou l'usage de communication radio "classique" n'est pas envisagée de manière prioritaire. En revanche, le développement de méthodes spécifiques de traitement de ces jeux de données non-idéaux, notamment des algorithmes automatiques de sélection et de pré-traitement des images, 
ainsi que l'optimisation de la chaîne de traitements photogrammétriques pour de gros jeux de données semblent très prometteurs pour améliorer à la fois la mise en œuvre de la méthode et la qualité des produits obtenus.

\section{Références}

Bogacki, M., Giersz, M., Patrycja, P.-G., Wieslaw, M., Misiewicz, K., 2010. Gps rtk mapping, kite aerial photogrammetry, geophysical survey and gis based analysis of surface artifact distribution at the pre-hispanic site of the castillo de huarmey, north coast of peru. Dans : Reuter, R. (Ed.), Remote Sensing for Science, Education, and Natural and Cultural Heritage. Paris, pp. 121-130.

Colomina, I., Molina, P., juin 2014. Unmanned aerial systems for photogrammetry and remote sensing : A review. ISPRS Journal of Photogrammetry and Remote Sensing, Volume 92, p. 79-97. 92, 79-97.

Crete, F., Dolmiere, T., Ladret, P., Nicolas, M., 2007. The blur effect : perception and estimation with a new no-reference perceptual blur metric. Vol. 6492. pp. 64920I-64920l-11.

El Maaoui, M. A., Felfoul, M. S., Boussema, M. R., Snane, M. H., 2012. Sediment yield from irregularly shaped gullies located on the fortuna lithologic formation in semi-arid area of tunisia. \{CATENA\} 93 (0), 97 - 104.

Fraser, W., Carlson, J., Duley, P., Holm, E., Patterson, D., 1999. Using kite-based aerial photography for conducting Adelie penguin censuses in Antarctica. WATERBIRDS 22 (3), 435440.

Harwin, S., Lucieer, A., 2012. Assessing the accuracy of georeferenced point clouds produced via multi-view stereopsis from unmanned aerial vehicle (uav) imagery. Remote Sensing 4 (6), 1573-1599.

Hassen, R., Wang, Z., Salama, M., July 2013. Image sharpness assessment based on local phase coherence. Image Processing, IEEE Transactions on 22 (7), 2798-2810.

Lowe, D. G., September 1999. Object recognition from local scale-invariant features. Dans : International Conference on Computer Vision. Corfu, Greece, pp. 1150-1157.

Marzolff, I., Ries, J., Albert, K., 18-20 September 2002, Bonn, Germany, 2002. Kite aerial photography for gully monitoring in sahelian landscapes. Dans: Proceedings of the Second Workshop of the EARSeL Special Interest Group on Remote Sensing for Developing Countries. Bonn, Germany, pp. 2-13.

Mekki, I., Albergel, J., Mechlia, N. B., Voltz, M., 2006. Assessment of overland flow variation and blue water production in a farmed semi-arid water harvesting catchment. Physics and Chemistry of the Earth, Parts A/B/C 31 (17), 1048 - 1061, <ce :title>Assessing Water Quality on Catchment Scale $</$ ce :title $>$.

Murray, J. C., Neal, M. J., Labrosse, F., MAR-APR 2013. Development and Deployment of an Intelligent Kite Aerial Photography Platform (iKAPP) for Site Surveying and Image Acquisition. JOURNAL OF FIELD ROBOTICS 30 (2), 288-307.

Pierrot-Deseilligny, M., Paparoditis, N., February 2006. A multiresolution and optimization-based image matching approach : An application to surface reconstruction from spot5-hrs stereo imagery. Dans : International Archives of Photogrammetry, Remote Sensing and Spatial Information Sciences. Vol. Vol. 36 (Part 1/W41). Ankara, Turkey.

Raclot, D., Albergel, J., 2006. Runoff and water erosion modelling using WEPP on a Mediterranean cultivated catchment. Physics and Chemistry of the Earth 31, 1038-1047.

Verhoeven, G. J. J., OCT-DEC 2009. Providing an Archaeological Bird's-eye View - an Overall Picture of Ground-based Means to Execute Low-altitude Aerial Photography (LAAP) in Archaeology. ARCHAEOLOGICAL PROSPECTION 16 (4), 233-249. 